\title{
Transient features in a Titan sea
}

\author{
J. D. Hofgartner ${ }^{1 \star}$, A. G. Hayes ${ }^{1}$, J. I. Lunine ${ }^{1}$, H. Zebker ${ }^{2}$, B. W. Stiles ${ }^{3}$, C. Sotin ${ }^{3}$, J. W. Barnes ${ }^{4}$, \\ E. P. Turtle ${ }^{5}$, K. H. Baines ${ }^{3}$, R. H. Brown ${ }^{6}$, B. J. Buratti ${ }^{3}$, R. N. Clark' ${ }^{7}$, P. Encrenaz ${ }^{8}$, R. D. Kirk ${ }^{9}$, \\ A. Le Gall ${ }^{10}$, R. M. Lopes ${ }^{3}$, R. D. Lorenz ${ }^{5}$, M. J. Malaska ${ }^{3}$, K. L. Mitchell ${ }^{3}$, P. D. Nicholson' ${ }^{1}$ P. Paillou ${ }^{11}$, \\ J. Radebaugh ${ }^{12}$, S. D. Wall ${ }^{3}$ and C. Wood ${ }^{13}$
}

Titan's surface-atmosphere system bears remarkable similarities to Earth's, the most striking being an active, global methane cycle akin to Earth's water cycle ${ }^{1,2}$. Like the hydrological cycle of Earth, Titan's seasonal methane cycle is driven by changes in the distribution of solar energy ${ }^{2}$. The Cassini spacecraft, which arrived at Saturn in 2004 in the midst of northern winter and southern summer, has observed surface changes, including shoreline recession, at Titan's south pole $\mathrm{e}^{3,4}$ and equator ${ }^{5}$. However, active surface processes have yet to be confirmed in the lakes and seas in Titan's north polar region ${ }^{6-8}$. As the 2017 northern summer solstice approaches, the onset of dynamic phenomena in this region is expected ${ }^{6,7,9-12}$. Here we present the discovery of bright features in recent Cassini RADAR data that appeared in Titan's northern sea, Ligeia Mare, in July 2013 and disappeared in subsequent observations. We suggest that these bright features are best explained by the occurrence of ephemeral phenomena such as surface waves, rising bubbles, and suspended or floating solids. We suggest that our observations are an initial glimpse of dynamic processes that are commencing in the northern lakes and seas as summer nears in the northern hemisphere.

Anomalous, bright features were detected in Titan's north polar sea, Ligeia Mare, by the Cassini Titan Radar Mapper ${ }^{13}$ (RADAR) during the T92 synthetic aperture radar (SAR) pass (Fig. 1). Three preceding SAR observations (T25, T29 and T64) and a subsequent low-resolution SAR observation (T95) did not detect the anomalous features. The faint, grey spots in the circle of the T95 image are consistent with the speckle noise in the surrounding sea region and thus are not anomalous. Radar backscatter above the noise floor, however, was also detected during preceding T91 radar scatterometry-mode observations $s^{13}$ but we argue that this signal may not have originated from the anomalies. Subsequent Visual and Infrared Mapping Spectrometer (VIMS) and Imaging Science Subsystem observations (T93 and T94) also did not detect the anomalies. These eight passes, constituting all of the highresolution observations up to the present of the region of the anomalous features, are shown in Fig. 1. In radar images, brightness is determined by the normalized radar cross-section (NRCS), the ratio of the radar energy backscattered to the receiver compared with that from an isotropic scatterer ${ }^{14}$. Dynamic processes such as waves ${ }^{7}$, suspended particles ${ }^{3}$, or bubbles ${ }^{15}$ increase the NRCS. Such phenomena have not been confirmed in Titan's northern lakes and seas, which have a dielectric constant that indicates a methane-ethane composition and surface height variations of less than $1 \mathrm{~mm}$ (ref. 8). The progressive seasonal increase in insolation that is occurring however has been predicted to power the onset of energetic processes ${ }^{6,7,9-12}$ and we argue that these anomalous features are the observation of transient features in the seas. The regional extent of the anomalous signal, which does not seem to derive from a single contiguous structure but rather from distinct features, is approximately $20 \mathrm{~km}$ by $20 \mathrm{~km}$. A higher-zoom image of the anomalous features is provided in the Supplementary Methods along with further discussion of their morphology. The image formed from the range/Doppler-processed, T91 scatterometrymode signal has noticeably more speckle and lower resolution than the other images because scatterometry-mode observations are not optimized for the formation of range/Doppler-processed images ${ }^{13}$. We argue that this image still contains credible signal despite the greater speckle.

Hypotheses for the anomalous features detected in the T92 observation are organized into the following three broad categories. Anomalies could arise from non-geophysical artefacts in the SAR data, permanent, geophysical structures that are detected when observed only with specific geometries, or transitory features that are the result of a surface transformation. We systematically evaluate each of these hypotheses in the following paragraphs.

The appearance of non-geophysical artefacts in SAR images is a familiar problem in radar remote sensing and common artefacts include ambiguities, scalloping, gain control, and edge effects ${ }^{14,16}$. Ambiguities result in a copy or 'ghost' of a region appearing offset in the range and/or azimuth directions. Range ambiguities occur when the radar instrument receives overlapping returns in the time domain from adjacent echo pulses whereas azimuth ambiguities arise from aliasing in the frequency domain of an echo. We found that there are no structures that could have resulted in bright range or azimuth ambiguities in the vicinity of the anomalous features. Nadir ambiguities, scalloping, and gain control effects are unlikely to create artefacts that are as spatially confined as the anomalies ${ }^{14}$. The anomalous features are surrounded by dark pixels, indicating that they are unlikely the result of an edge effect. Thus, the anomalies are not considered to be standard SAR image artefacts. We provide more detailed arguments in the Supplementary Methods to support our conclusion that a SAR artefact is not the explanation for the anomalous features.

\footnotetext{
${ }^{1}$ Department of Astronomy, Cornell University, Ithaca, New York 14853, USA, ${ }^{2}$ Department of Electrical Engineering, Stanford University, Stanford, California 94305-2215, USA, ${ }^{3}$ Jet Propulsion Laboratory, Pasadena, California 91109, USA, ${ }^{4}$ Department of Physics, University of Idaho, Moscow, Idaho 83844-0903, USA, ${ }^{5} \mathrm{JHU}$ Applied Physics Lab, Laurel, Maryland 20723, USA, ${ }^{6}$ Lunar and Planetary Laboratory, University of Arizona, Tucson, Arizona 85721, USA, 7 USGS Denver Federal Center, Denver, Colorado 80225-0046, USA, ${ }^{8}$ Observatoire de Paris, Paris 75014 , France, ${ }^{9}$ USGS Astrogeology Center, Flagstaff, Arizona 86001, USA, ${ }^{10}$ LATMOS-UVSQ, Paris 78280, France, ${ }^{11}$ University of Bordeaux, Bordeaux 33271, France, ${ }^{12}$ Department of Geological Sciences, Brigham Young University, Provo, Utah 84602, USA, ${ }^{13}$ Planetary Science Institute, Tucson, Arizona 85721, USA. *e-mail: jhofgartner@astro.cornell.edu
} 


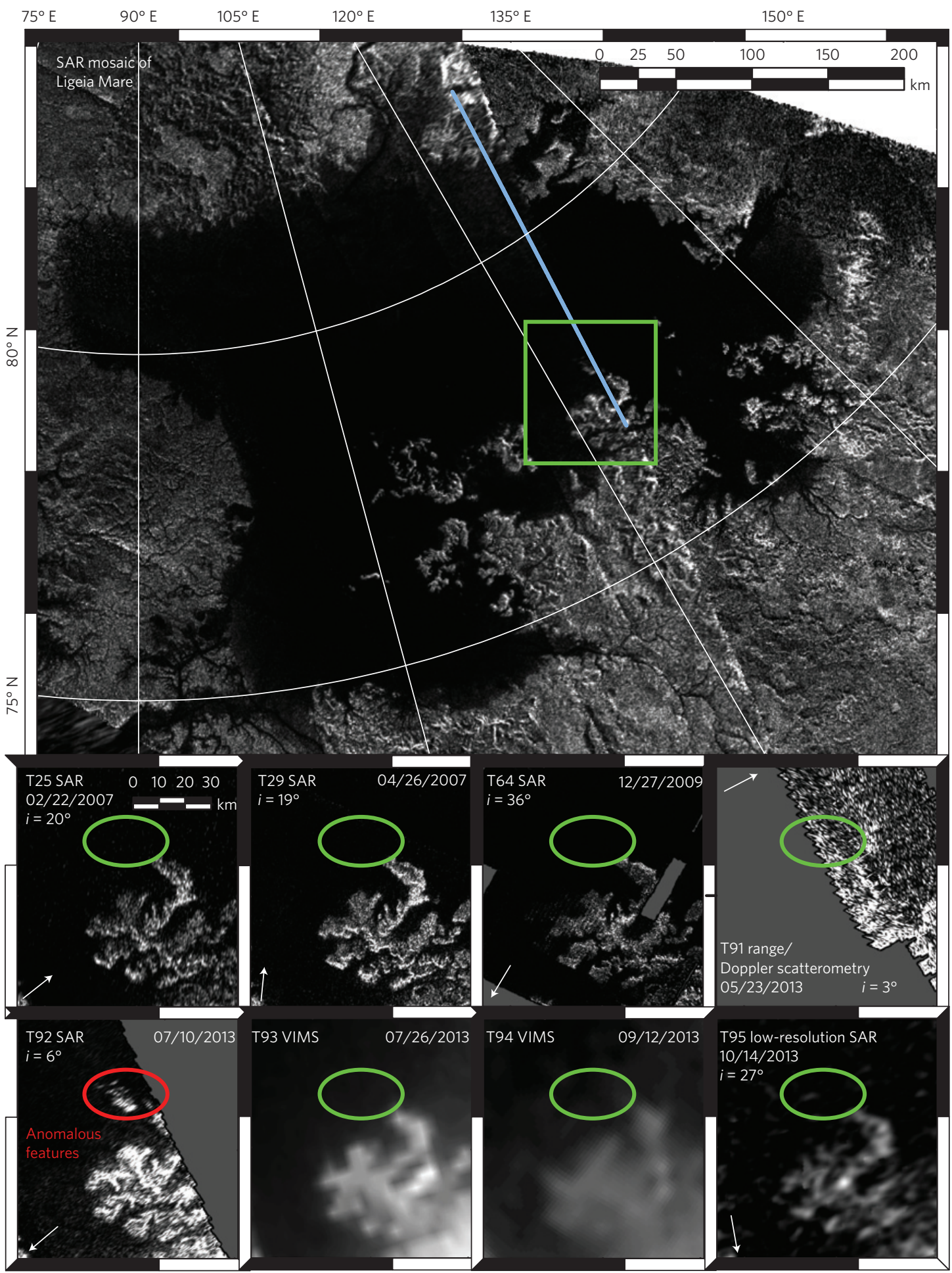

Figure 1| Titan's Ligeia Mare and high-resolution Cassini observations of the region of the anomalous features (green outlines). In the T92 image, anomalous, bright features (circled in red) are observed at $78^{\circ} \mathrm{N}, 123^{\circ} \mathrm{E}$ that are not seen in any of the other SAR or VIMS images. Similarly sized, nearby peninsulas (bright region at the bottom right), however, were consistently detected. The transient anomalies were probably not present during the T91 scatterometry-mode observation. Pixel brightness is linearly related to normalized radar cross-section. Green rectangle indicates the extent of the high-resolution images, and green ovals correspond to the area circled in red. White arrows in radar images indicate the radar illumination direction. The blue line indicates the transect for Fig. 3.

For Cassini RADAR measurements of a permanent, geophysical structure on Titan, the angle of incidence is the dominant geometrical parameter for the measured NRCS. These two variables are inversely correlated; that is, increasing the angle of incidence decreases the NRCS (ref. 14). Figure 2 is a plot of the NRCS from the region of the anomalous features as a function of incidence angle. Only the T91 and T92 observations, at incidence angles of 3 and 6 degrees respectively from the surface normal (black circles), measured radar backscatter above the noise floor (red triangles). Thus, any model for the anomalous features as permanent, static 


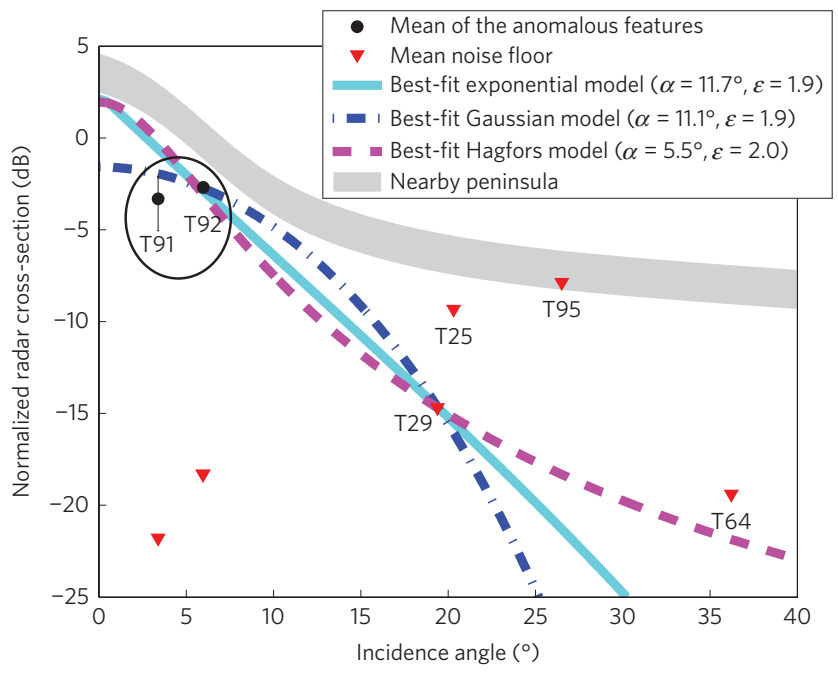

Figure 2 | Normalized radar cross-section of the region of the anomalous features as a function of incidence angle. Only the T91 and T92

observations, at incidence angles of 3.4 and 6.0 degrees respectively (black circles), measured radar backscatter above the noise floor (red triangles). Quasi-specular models are ruled out to $88 \%$ confidence because, as shown, most cannot simultaneously satisfy the shallow slope between the T91 and T92 observations and the upper limits at higher incidence angles. The grey shaded region shows the behaviour of the nearby peninsulas, which is consistent with quasi-specular plus diffuse scattering. Error bars show one-sigma confidence.

structures must be consistent with the T91 and T92 measurements and stay below the noise floor values of the higher incidence angle observations (otherwise the anomalous features would have been detected in those observations as well). Empirically, all terrains on Titan can be well fitted by combined quasi-specular and diffuse backscatter models ${ }^{17,18}$, including the nearby peninsulas (Supplementary Methods), visible towards the lower right in the zoom panels of Fig. 1. We compared the observations of the anomalies with a suite of quasi-specular plus diffuse backscatter models and found that this class of models for a permanent structure can be ruled out to $88 \%$ confidence (Methods). The best-fit models are plotted in Fig. 2 and their parameters are given in the legend. We also considered models for submerged seamounts, using constraints for the surface roughness and dielectric constant of Ligeia Mare derived from recent analyses of the nadir ( 0 degrees incidence) signal in the T91 observation ${ }^{8,19}$ and found that these models are also ruled out to $88 \%$ confidence (Methods). We note that it is the combination of the small likelihood that the NRCS at 3 degrees is larger than at 6 degrees with the low upper limits at higher incidence angles that inhibits the models from fitting the observations.

We point out that the NRCS upper limits for incidence angles of greater than 15 degrees require that permanent models for the anomalous features not exhibit any appreciable diffuse radar scattering. An absence of diffuse radar scattering however is discordant with the general conclusion, not only from the Cassini spacecraft's $2.2 \mathrm{~cm}$ wavelength observations but also from the $3.5 \mathrm{~cm}$ and $12.6 \mathrm{~cm}$ Earth-based observations, that radar scattering on Titan is dominated by diffuse backscatter ${ }^{17,18,20,21}$. The nearby peninsulas, for example, exhibit significant diffuse backscatter, as shown by the grey shaded region in Fig. 2 . Thus, the set of models that has a $12 \%$ chance of corresponding to the data has the important caveat that none of those models scatters radar waves diffusely, a behaviour that is dissimilar from all other terrains on Titan. Therefore, we also consider those models implausible.

The T91 and T92 NRCS profiles along a transect of Titan that crosses Ligeia Mare and includes the region of the anomalous

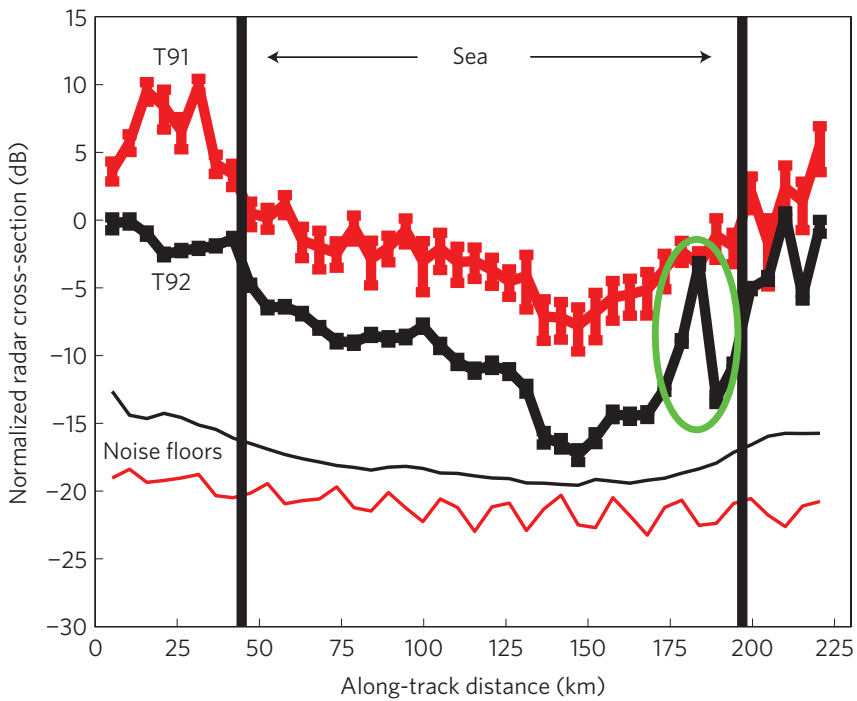

Figure $\mathbf{3}$ | Normalized radar cross-section profiles along a transect of Titan that crosses Ligeia Mare including the region of the anomalous features. The correlation of the profiles suggests that the signal in the T91 image is valid. At the anomalous features (green oval), the T92 profile exhibits a large spike but no similar spike is observed in the T91 profile. Incidence angle increases from $3.3^{\circ}-3.5^{\circ}$ and $4.6^{\circ}-6.2^{\circ}$ for the T91 and T92 observations respectively. The blue line in Fig. 1 indicates the centre of the transect. The error bars show the one-sigma confidence.

features are plotted in Fig. 3. These profiles, which are both above their respective noise floors, are correlated and the lesser T92 NRCS, relative to that of T91, is due to its greater incidence angle. This behaviour is consistent, for example, with the radar transmitting through the liquid and scattering off the seabed as claimed by other analyses $s^{3,19,22,23}$. In the region of the anomalies, however, the T92 profile exhibits a large spike in NRCS but no similar anomalous spike is observed in the T91 profile. Therefore, we conclude that the anomalous features were not present at the time of the T91 observation.

Transitory hypotheses envisage that a transformation occurred before the discovery of the anomalous features and that their detection thus depends primarily on the timing of the observation. The anomalies were not detected in three observations before 2013, were detected in the T92 observation on 10 July 2013 and then not detected in three subsequent observations (Fig. 1). A signal was detected in the T91 observation on 23 May 2013 but as previously discussed, the transient anomalies probably were not present during this observation. Therefore, the evolution of the anomalous features seems to have included a reversion after the T92 pass and we do not consider further hypotheses that predict the formation of a new permanent structure, such as an island through cryovolcanism and restrict further discussion to hypotheses for ephemeral features. From recent analysis of the nadir signal in the T91 observation ${ }^{19}$, the absorption of the radar energy as it propagates through the sea is constrained to be small. Thus, variations in sea level should not strongly influence the measured NRCS and are unlikely to explain the transient anomalies. With the exception of the T25 and T29 passes, all of the passes occurred at the same true orbital anomaly and thus hypotheses that depend on Titan's orbit around Saturn, such as tides, are also not considered further.

The remaining transient hypotheses include waves, rising bubbles, and suspended and/or floating solids. The data do not permit us to further discard any of these hypotheses with confidence. Titan's northern hemisphere is transitioning from vernal equinox (August 2009) to summer solstice (May 2017) and it is plausible that the anomalous, transient features are an expression 
of the changing seasons. Waves were/are expected to form and become detectable as wind speeds in the northern hemisphere climb with the approach of summer ${ }^{6,7,9,10}$. Thermal perturbations could lead to the exsolution of gases from the liquid and/or sea floor that form bubbles and buoyantly rise to the surface. Polyacetylene and other low-density solids could be suspended in the sea ${ }^{24}$ much like silt in a terrestrial delta (backscatter variation, possibly from a transient surface layer with distinct dielectric properties, has been previously observed near an estuary of Kraken Mare ${ }^{3}$ ) and it has been predicted that sunken solids formed from a winter freeze could become buoyant with the onset of warmer temperatures $^{12}$. This discussion of seasonal mechanisms is not inclusive and all of the remaining hypotheses may have additional plausible mechanisms.

Some of the ephemeral phenomena cited above as causes of the transient radar signature may be stimulated or enhanced by regional meteorological phenomena, such as wind or rain. Although Cassini did not detect any clouds during the T92 pass, the geometry was rather unfavourable for detection above the site of the anomalies; we note, however, that during the T93 pass VIMS detected a cloud, approximately $100 \mathrm{~km}$ in diameter, about $350 \mathrm{~km}$ from the region of the transients.

\section{Methods}

Mask. To determine the mean NRCS of the regions of the anomalous features and thus produce the plot in Fig. 2, it was necessary to define a mask that encompassed exclusively the regions of the anomalous features. We considered a zone that, from visual inspection, included all pixels of the anomalous features as well as some sea pixels but none of the shore. The mask for the anomalies was defined as all pixels in this zone with an NRCS in the T92 image of greater than 0.25 and was used to determine the average characteristics in each observation of the region of the anomalies. The cutoff of 0.25 was selected to eliminate $>99 \%$ of the sea pixels, from analysis of their backscatter distribution, but retain most of the pixels of the anomalous features. As radar measurements of any feature will have an exponential distribution (speckle), a threshold that removes the lower end of the distribution biases the mean NRCS towards a higher value. We found that the cutoff only minimally biased the T92 NRCS and did not significantly affect the results.

Modelling. We considered three classes of quasi-specular models: exponential, Gaussian and Hagfors ${ }^{18}$. To test whether the data are consistent with these models, we simulated the T91 and T92 observations by randomly generating NRCS values such that they followed a normal distribution, with the mean and standard deviation given by the observed NRCS and error. We then checked whether any models fit the two simulated backscatter measurements and remained below the upper limits at higher incidence angles. The exponential, Gaussian and Hagfors models failed to fit the data in $>99 \%, 88 \%$ and $>99 \%$ of the simulations respectively. The best-fit models are plotted in Fig. 2 and their root-mean-square roughnesses and effective dielectric constants are given in the legend. The success rate of Gaussian models was greater than exponential and Hagfors models because they predict a shallower gradient in NRCS at the lowest incidence angles (less than about 10 degrees) and a steeper gradient at higher incidence angles. This is consistent with the measured NRCS, which is approximately flat for the lowest incidence angles but significantly reduced by about 20 degrees incidence.

Including the additional physics of refraction and loss due to reflection at the atmosphere-sea interface ${ }^{23}$, we followed the same prescription as above to test models for submerged seamounts. We used the recently measured index of refraction for Ligeia Mare to calculate the refracted incidence angles of the $\operatorname{radar}^{19}$ and considered a perfectly flat surface for Ligeia Mare, consistent with recent measurements ${ }^{8}$, when calculating the Fresnel transmission coefficients.

\section{Received 13 March 2014; accepted 20 May 2014;} published online 22 June 2014

\section{References}

1. Atreya, S. K. et al. Titan's methane cycle. Planet. Space Sci. 54, 1177-1187 (2006).

2. Lunine, J. I. \& Atreya, S. K. The methane cycle on Titan. Nature Geosci. 1, $159-164$ (2008)
3. Hayes, A. G. et al. Transient surface liquid in Titan's polar regions from Cassini. Icarus 211, 655-671 (2011).

4. Turtle, E. P., Perry, J. E., Hayes, A. G. \& McEwen, A. S. Shoreline retreat at Titan's Ontario Lacus and Arrakis Planitia from Cassini Imaging Science Subsystem observations. Icarus 212, 957-959 (2011).

5. Turtle, E. P. et al. Rapid and extensive surface changes near Titan's equator: Evidence of April showers. Science 331, 1414-1417 (2011).

6. Lorenz, R. D., Newman, C. \& Lunine, J. I. Threshold of wave generation on Titan's lakes and seas: Effect of viscosity and implications for Cassini observations. Icarus 207, 932-937 (2010).

7. Hayes, A. G. et al. Wind driven capillary-gravity waves on Titan's lakes: Hard to detect or non-existent? Icarus 225, 403-412 (2013).

8. Zebker, H. A. et al. Surface of Ligeia Mare, Titan, from Cassini altimeter and radiometer analysis. Geophys. Res. Lett. 41, 308-313 (2013).

9. Tokano, T. Limnological structure of Titan's hydrocarbon lakes and its astrobiological implication. Astrobiology 9, 147-164 (2009).

10. Tokano, T. Are tropical cyclones possible over Titan's seas? Icarus 223, 766-774 (2013)

11. Roe, H. G. \& Grundy, W.M. Buoyancy of ice in the $\mathrm{CH}_{4}-\mathrm{N}_{2}$ system. Icarus 219, 733-736 (2012)

12. Hofgartner, J. D. \& Lunine, J. I. Does ice float in Titan's lakes and seas? Icarus 223, 628-631 (2013)

13. Elachi, C. et al. RADAR: The Cassini Titan radar mapper. Space Sci. Rev. 115, 71-110 (2004).

14. Elachi, C. \& van Zyl, J. Introduction to the Physics and Techniques of Remote Sensing (Wiley, 2006).

15. Engram, M., Anthony, K. W., Meyer, F. J. \& Grosse, G. Synthetic aperture radar (SAR) backscatter response from methane ebullition bubbles trapped by thermokarst lake ice. Can. J. Remote Sens. 38, 667-682 (2012).

16. Stiles, B. W. et al. Ground processing of Cassini RADAR imagery of Titan. (Proc. IEEE Conference on RADAR, 2006).

17. Wye, L. C. et al. Electrical properties of Titan's surface from Cassini RADAR scatterometer measurements. Icarus 188, 367-385 (2007).

18. Wye, L. C. Radar scattering from Titan and Saturn's icy satellites using the Cassini spacecraft. PhD thesis, Stanford University, Faculty of Engineering, 316 (2011).

19. Mastroguiseppe, M. et al. The bathymetry of a Titan sea. Geophys. Res. Lett. 41, 1432-1437 (2014).

20. Muhleman, D. O., Grossman, A. W. \& Butler, B. J. Radar investigations of Mars, Mercury, and Titan. Annu. Rev. Earth Planet. Sci. 23, 337-374 (1995).

21. Campbell, D. B., Black, G. J., Carter, L. M. \& Ostro, S. J. Radar evidence for liquid surfaces on Titan. Science 302, 431-434 (2003).

22. Hayes, A. G. et al. Hydrocarbon lakes on Titan: Distribution and interaction with a porous regolith. Geophys. Res. Lett. 35, L09204 (2008).

23. Hayes, A. G. et al. Bathymetry and absorptivity of Titan's Ontario Lacus. J. Geophys. Res. 115, E09009 (2010).

24. Chien, J. C. W. Polyacetylene Chemistry, Physics and Material Science (Academic Press, 1984).

\section{Acknowledgements}

J.D.H. gratefully acknowledges the Cassini RADAR and VIMS Teams for the data and the opportunity to lead the analysis and the Cassini Project and Natural Sciences and Engineering Research Council of Canada, Post Graduate Scholarship Program for financial support. A.G.H. was partially supported by NASA grant NNX13AG03G A portion of this work was performed at the Jet Propulsion Laboratory, California Institute of Technology under a contract with the National Aeronautics and Space Administration.

\section{Author contributions}

J.D.H. led the analysis and writing of the letter. A.G.H. and J.I.L. worked closely with J.D.H. on all aspects of the analysis and writing. H.Z. worked closely with J.D.H. on the NRCS analysis. B.W.S. contributed the SAR processing and artefact analysis and that section of the letter. C.S., J.W.B. and E.P.T. contributed to the VIMS and Imaging Science Subsystem analysis and the cloud discussion. All authors contributed to the data acquisition and discussions.

\section{Additional information}

Supplementary information is available in the online version of the paper. Reprints and permissions information is available online at www.nature.com/reprints. Correspondence and requests for materials should be addressed to J.D.H

\section{Competing financial interests}

The authors declare no competing financial interests. 